\title{
INVERSIVE PLANES OF EVEN ORDER
}

\author{
BY PETER DEMBOWSKI
}

Communicated by A. M. Gleason, June 10, 1962

1. Results. An inversive plane is an incidence structure of points and circles satisfying the following axioms:

I. Three distinct points are connected by exactly one circle.

II. If $P, Q$ are two points and $c$ a circle through $P$ but not $Q$, then there is exactly one circle $c^{\prime}$ through $P$ and $Q$ such that $c \cap c^{\prime}=\{P\}$.

III. There are at least two circles. Every circle has at least three points.

For any point $P$ of the inversive plane $\Im$, the points $\neq P$ and the circles through $P$ form an affine plane $\mathfrak{A}(P)$. If $\Im$ is finite, all these affine planes have the same order (number of points per line); this integer is also termed the order of $\Im$. An inversive plane of order $n$ consists of $n^{2}+1$ points and $n\left(n^{2}+1\right)$ circles; every circle contains $n+1$ points, and any two points are connected by $n+1$ circles.

Let $\mathfrak{P}$ be a projective space of dimension $d>1$ (we shall only be concerned with $d=2,3$, and we do not assume the theorem of Desargues if $d=2$ ). A point set $\mathbb{C}$ in $\mathfrak{B}$ is called an ovoid if

$\mathrm{I}^{\prime}$. Any straight line of $\mathfrak{B}$ meets $\mathbb{C}$ in at most two points;

$\mathrm{II}^{\prime}$. For any $P \in \mathfrak{C}$, the union of all lines $x$ with $x \cap \mathfrak{E}=\{P\}$ is a hyperplane.

(This is called the tangent hyperplane to $\mathbb{C}$ in $P$.) It is straightforward to prove that the points and the nontrivial plane sections of an ovoid in a three-dimensional projective space form an inversive plane. The purpose of the present note is the announcement, and an outline of proof, of the following partial converse:

THEOREM 1. Every inversive plane of even order $n$ is isomorphic to the system of points and plane sections of an ovoid in a three-dimensional projective space over $\mathrm{GF}(n)$.

We list three immediate corollaries: If $\Im$ is an inversive plane of even order $n$, then (i) $n$ is a power of 2 , (ii) for any $P \in \Im$, the affine plane $\mathfrak{A}(P)$ is desarguesian, and (iii) $\Im$ satisfies the bundle theorem ("Büschelsatz," cf., e.g., [2]).

The proof of Theorem 1, to be outlined in $\$ 2$ below, shows also that every automorphism (incidence preserving permutation) of an inversive plane of even order can be extended to a collineation, leaving the representing ovoid invariant, of the appropriate projective space. Together with recent results of Tits [9], [10], this leads to a complete 
classification of all inversive planes of even order whose automorphism group is at least doubly transitive.

An inversive plane represented by points and plane sections of a nonruled quadric can be internally characterized by the validity of Miquel's theorem, see [2], [5], [11], and will therefore be called miquelian.

Theorem 2. An inversive plane of even order admits a triply transitive automorphism group if, and only if, it is miquelian.

The only known non-miquelian finite inversive planes are of order $n=2^{2 m-1}, m>1$; the corresponding ovoids are the point sets defined by

$$
z=x y+x^{\omega+2}+y^{\omega}
$$

in a suitable affine space over $\mathrm{GF}(n)$, with the plane at infinity a tangent plane; here $\omega$ is the automorphism $x \rightarrow x^{2^{m}}$ of $\mathrm{GF}(n)$, see [8], [10]. The automorphism groups of these inversive planes are essentially the Suzuki groups $S z(n)$, see [6], [10]. In view of another result of Tits, announced in [9], we have

THEOREM 3. The full automorphism group of an inversive plane of even order is doubly, but not triply transitive if, and only if, the plane is represented by an ovoid of type (*).

Thus the known inversive planes of even order are precisely the doubly transitive ones.

2. Proof of Theorem 1. Let $\Im$ be an inversive plane of even order $n$. We define a new incidence structure $\mathfrak{P}$ of points and planes as follows: Points are (a) the points of $\Im$ (real points), and (b) the circles of $\Im$ (ideal points; if $c$ is a circle, the corresponding ideal point will be denoted by $c^{\pi}$ ). Planes are (a) the circles of $\Im$ (real planes), and (b) the points of $\Im$ (ideal planes; if $P$ is a point, the corresponding ideal plane is denoted by $P^{\pi}$ ). Incidence in $\Re$, denoted by the symbol $I$, is defined among real points and planes as in $\Im$, and otherwise by the following rules:

(i) $P I Q^{\pi} \Leftrightarrow P=Q$,

(ii) $c^{\pi} I Q^{\pi} \Leftrightarrow c I Q$,

(iii) $c^{\pi} I d \Leftrightarrow c=d$ or $|c \cap d|=1$.

The incidence structure $\mathfrak{B}$ so defined consists of $(n+1)\left(n^{2}+1\right)$ points and equally many planes. In fact, $\mathfrak{P}$ is self-dual, for the mapping $P \rightarrow P^{\pi}, c \rightarrow c^{\pi}$ can be extended in a natural way to a null polarity of $\mathfrak{B}$. In view of a characterization of finite projective spaces given in [3] (see also [12]), the condition that $\mathfrak{P}$ be a projective space is equivalent to the following conditions: 
(A) Every plane is incident with $n^{2}+n+1$ points.

(B) Two distinct points are connected by $n+1$ planes.

(C) The intersection of all planes containing two distinct points consists of $n+1$ points.

In order to prove Theorem 1 it suffices to verify conditions (A)(C) for the incidence structure $\mathfrak{B}$ defined above. The complete proof of this requires the separate treatment of several different cases and will not be given here. We carry the argument only to a point from which the remainder is a matter of straightforward checking.

We call two circles of $\Im$ tangent if they have precisely one common point.

(1) If $P, c$ is a nonincident point-circle pair in $\Im$, then the circles through $P$ which are tangent to $c$ are precisely the $n+1$ circles connecting $P$ with some other point.

For in the projective plane corresponding to the affine plane $\mathfrak{A}(P)$, the circle $c$ is an ovoid, not meeting the line at infinity, and the circles tangent to $c$ through $P$ are the tangent lines of this ovoid. It is well known (see, e.g., [5]) that the tangents of an ovoid in a projective plane of even order are exactly the lines through some point $Q$. As $Q$ is not on the line at infinity, we have $P \neq Q$, and (1) follows. We note an immediate corollary:

(2) Three mutually tangent circles of $\Im$ pass through one point.

Next, we prove two other consequences of (1).

(3) If $P, Q, R$ are three distinct points of $\Im$, then there is exactly one circle $c$ through $R$ which is tangent to each of the $n+1$ circles through $P$ and $Q$.

The uniqueness of $c$ follows from (2): two distinct circles $c, c^{\prime}$ of the required sort would be tangent to each other (since they are both tangent to $P Q R$ ) and to any circle $\neq P Q R$ through $P, Q$.

Existence. ${ }^{1}$ Let $x$ be any one of the $n^{2}-1$ circles which pass through $R$ but not through $P$, and denote by $Q(x)$ the second common point of all the circles through $P$ and tangent to $x$, given by (1). By the uniqueness already established, the mapping $x \rightarrow Q(x)$ is one-one. Hence $Q(x)$ ranges over all the $n^{2}-1$ points different from $P$ and $R$, and there is an $x$ for which $Q(x)=Q$.

(4) If $c, d$ are two disjoint circles of $\Im$, then the common tangents of $c$ and $d$ are exactly the $n+1$ circles through two uniquely determined points $P$ and $Q$.

Proof. Through any point $X$ on $c$ there is at most one common

\footnotetext{
${ }^{1}$ My original proof of this part was more complicated. For the present improvement I am indebted to D. R. Hughes.
} 
tangent to $c$ and $d$, by (2). As the number $n+1$ of points on $d$ is odd, and since every point on $d$ is on some tangent to $c$ through $X$ (Axiom II), one of these tangents must also be tangent to $d$; hence there is precisely one common tangent through $X$. This shows that $n+1$ is the total number of common tangents of $c$ and $d$, and therefore there is at most one point pair $P, Q$ of the required kind. The existence of $P, Q$ is then proved by an argument similar to that of the corresponding part of the proof of (3).

From here on, the verification of conditions (A)-(C) presents no more difficulties. Instead of giving the details, we only say what the straight lines of $\Re$ are: An external line consists of $n+1$ ideal points $x^{\pi}$ corresponding to the circles $x$ through two real points $P, Q$; and it is contained in the two ideal planes $P^{\pi}, Q^{\pi}$ and the $n-1$ real planes [(3) is used] tangent to all circles through $P$ and $Q$. A tangent line consists of one real point $P$ and $n$ ideal points corresponding to a maximal set of mutually tangent circles through $P$; it is contained in these real planes and the ideal plane $P^{\pi}$. And a secant line consists of two real points $P, Q$ and the $n-1$ ideal points $x^{\pi}$ corresponding to the circles tangent to every circle through $P$ and $Q$; it is contained in the $n+1$ real planes through $P$ and $Q$.

It is clear from these remarks that the set of real points is an ovoid in the projective space $\mathfrak{B}$, so that Theorem 1 is established. The definitions (i)-(iii) of incidence in $\mathfrak{P}$ show that any automorphism of $\Im$ can be extended to a collineation of $\mathfrak{P}$, so that Theorems 2 and 3 may be deduced as indicated in $\$ 1$.

We conclude with a few remarks on the hypotheses of Theorem 1. There are infinite inversive planes which cannot be represented by ovoids [4]; hence the finiteness condition in Theorem 1 is essential. It is, however, an open question whether Theorem 1 is also true for inversive planes of odd order (by a result of Barlotti [1], such a theorem would imply that all inversive planes of odd order are miquelian). The idea of the proof of Theorem 1 can be utilized to give necessary and sufficient conditions for an arbitrary finite inversive plane $\Im$ to be representable by an ovoid: $\Im$ must admit an "orthogonality relation" (of a kind similar to that discussed in [2]) among its circles. It would lead too far to give more details here; a complete discussion, together with a full proof of Theorem 1 , will be given elsewhere.

\section{REFERENCES}

1. A. Barlotti, Un' estensione del teorema di Segre-Kustaanheimo, Boll. Un. Mat. Ital. 10 (1955), 498-506. 
2. W. Benz, Ueber Möbiusebenen. Ein Bericht. Jber. Deutsch. Math.-Verein. 63 (1960), 1-27.

3. P. Dembowski and A. Wagner, Some characterizations of finite projective spaces, Arch. Math. 11 (1960), 465-469.

4. G. Ewald, Beispiel einer Möbiusebene mit nichtisomorphen affinen Unterebenen, Arch. Math. 11 (1960), 146-150.

5. A. J. Hoffman, Chains in the projective line, Duke Math. J. 18 (1951), 827-830.

6. D. R. Hughes, Combinatorial analysis. t-designs and permutation groups, 1960 Institute on Finite Groups, Proc. Sympos. Pure Math. Vol. 6, pp. 39-41, Amer. Math. Soc., Providence, R. I., 1962.

7. B. Qvist, Some remarks concerning curves of the second degree in a finite plane, Ann. Acad. Sci. Fenn. 134 (1952).

8. B. Segre, On complete caps and ovaloids in three-dimensional Galois spaces of characteristic two, Acta Arith. 5 (1959), 315-332.

9. J. Tits, Ovoïdes a translations, Rend. Mat. e Appl. 21 (1962), 37-59.

10. - - Ovoïdes et groupes de Suzuki, Arch. Math. 13 (1962), 187-198.

11. B. L. van der Waerden und L. J. Smid, Eine Axiomatik der Kreisgeometrie und der Laguerregeometrie, Math. Ann. 110 (1935), 753-776.

12. A. Winternitz, Zur Begründung der projektiven Geometrie. Einfïhrung idealer Elemente, unabhängig von der Anordnung, Ann. of Math. (2) 41 (1940), 365-390.

UNIVERSITÄT FRANKFURT AM MAIN AND

Queen Mary College, London

\title{
MICROBUNDLES ARE FIBRE BUNDLES ${ }^{1}$
}

\author{
BY J. M. KISTER
}

Communicated by Deane Montgomery, June 10, 1963

Introduction. In [1], Milnor develops a theory for structures, known as microbundles which generalize vector bundles. It is shown there that this is a proper generalization; that some microbundles cannot be derived from any vector bundle. It is then possible, for instance, to find a substitute (tangent microbundle) for the tangent bundle over a manifold $M$ even though $M$ admits no differential structure.

A well-known and more general class of structures than vector bundles (but less general than microbundles) is the class of fibre bundles with a Euclidean fibre and structural group the originpreserving homeomorphisms of Euclidean space topologized by the compact-open topology (cf. [2]). In this note such structures will be

1 This work was supported by a grant from the Institute for Advanced Study and by NSF grant G-24156. 